\title{
Editorial
}

Gene Therapy (2004) 11, 343. doi:10.1038/sj.gt.3302212

\section{Orthopaedics: gene therapy's dark horse}

At first sight, orthopaedics and gene therapy appear to have little to offer each other. Genetic abnormalities of the skeletal system are rare and complex, few orthopaedic disorders are life threatening, and the adjective 'orthopaedic' is more often coupled to 'surgery' than 'gene'. So why an entire issue of Gene Therapy devoted to orthopaedics? Orthopaedic conditions provide a rich, albeit underappreciated, vein of opportunities for genetic intervention. Moreover, several applications promise success using existing technologies and may therefore be among the earliest gene therapies to establish a niche in clinical practice.

Tissue repair is a case in point. Many orthopaedic tissues, including cartilage, the anterior cruciate ligament and the intervertebral disc, do not heal well after injury. Even bone, normally capable of rapid, scar-less regeneration, can be problematic. Recent research has identified numerous growth factors and other gene products with the ability to promote the regeneration of these tissues, but their clinical use is hindered by delivery problems. In principle, these can be overcome by delivering the relevant cDNAs. The advantages of gene delivery include its ability to establish a local, endogenous synthesis of authentically processed material at the site of injury. Moreover, for tissue repair, long-term gene expression is neither necessary nor desired. Even with first-generation adenovirus vectors, the duration and level of transgene expression are sufficient for many purposes, at least in animal models. Such applications are well represented in this issue, which contains stateof-the-art reviews on progress in adapting gene transfer for healing bone, cartilage, ligaments and tendons, as well as improving the outcome of spinal fusions.

Chronic diseases constitute another group of promising orthopaedic targets. Most progress has been achieved with the genetic treatment of arthritis and disc degeneration, both reviewed here. Indeed, the only clinical trials yet initiated in the orthopaedic area involve gene transfer to joints. These trials reflect the promise of gene transfer to synovium as a platform technology for the treatment of arthritis and other joint disorders. As arthritis and disc degeneration are so prevalent, costly, painful and resistant to therapy, new strategies for treatment are especially welcome. As with tissue repair, gene therapy approaches are not based on compensating for genetic defects, but on the targeted, efficient delivery of therapeutic gene products to sites of disease activity. These principles can also be applied to aseptic loosening, an interesting iatrogenic condition that occurs through resorption of bone around prosthetic joints. Osteoporosis, another chronic orthopaedic condition of enormous socioeconomic importance, is an additional attractive, but ignored, target for gene therapy.

Genetic diseases of the skeletal system have also received scant attention. This partly reflects their rarity and the enormous technological problems many of them present. Dominant-negative mutations are common, and genetic therapies may need to be implemented very early, before aberrant development has progressed beyond the point of ready correction. Osteogenesis imperfecta, commonly known as 'brittle bone disease', reviewed here, has been the focus of most gene therapy research in this area.

Orthopaedic tumors, although also rare, are frequently devastating, as they often incur loss of life or limb in children. Cancer gene therapy is a thriving field of research, but it has not yet included orthopaedic tumors within its remit. The recent loss of a cousin to Ewing's sarcoma has alerted me to the urgent need for improved treatments for orthopaedic malignancies of this type. The absence of a review on orthopaedic cancers in this edition reflects the unfortunate neglect of this area.

Thus orthopaedic gene therapy is not the oxymoron it might at first seem. Genetic therapies promise to impact many areas of orthopaedics, including sports medicine, spine, joint replacement, trauma, arthritis and so forth. Orthopaedics, for its part, stands to provide gene therapy with some of its first widespread clinical successes. The first decade of this millennium has been officially designated the 'Bone and Joint Decade', a fitting backdrop to the advances described in this special issue of Gene Therapy.

$\mathrm{CH}$ Evans Center for Molecular Orthopaedics, Harvard Medical School, USA 